\title{
Phase contrast surface mode resonance microscopy
}

\author{
S. Herminghaus, C. Bechinger, W. Petersen, P. Leiderer \\ Universität Konstanz, Fakultät für Physik, Universitätsstrasse 10, D-78434 Konstanz, Germany
}

Received 10 June 1994; revised manuscript received 28 July 1994

\begin{abstract}
A simple method is described which allows to obtain a phase sensitive image in surface mode resonance microscopy. It exploits the fact that surface mode resonances couple only to one direction of polarization ( $s$ or $p$ ) so that the other direction ( $p$ or s) can be used as a reference wave. Using a Soleil-Babinet compensator and a polarizer, the two waves can be phase shifted with respect to each other and superimposed before they are imaged onto a camera chip. The method is demonstrated experimentally. Possible applications are discussed, especially for the field of nonlinear optics of thin films and surfaces, where phase information is particularly desirable.
\end{abstract}

\section{Introduction}

Surfaces and thin films can be sensitively characterized by optical excitation and detection of their optical eigenmodes. The electromagnetic field which accompanies these surface modes is concentrated closely to the surface, which results in a high field strength and thus a high sensitivity of the surface mode phase velocity on the physics of the system in which they propagate. This has been utilized for many years in attenuated total reflectance (ATR) spectroscopy of a large variety of physical systems, ranging from metal surfaces [1-6] and dielectric films [7-10] to complicated objects like polymer [11-13] and LangmuirBlodgett films [14], organic crystals [15] or biologically relevant systems $[16,17]$.

In general, ATR spectroscopy proceeds as follows [18]. A monochromatic light beam illuminates the system to be investigated, using a suitable coupling device (a prism or grating, see below). Every optical surface mode of the system has a characteristic dispersion and thus a characteristic wave number at the frequency of the incoming light. When the in-plane wave number of the incoming light matches the wave number of the surface mode, the latter is strongly excited at expense of the reflected intensity. One thus obtains dips in the reflected intensity at particular angles of incidence, each of which corresponds to a surface mode.

The (approximately) Lorentzian shape of the individual dips can be understood in terms of an interference between two possibilities for the photon to be reflected from the the system under consideration. Either the photon is 'directly' reflected by the discontinuity or it is converted into a virtual surface mode

quantum which then decays back into a photon leaving the surface. This picture leads to the following expression for the complex reflectivity amplitude [19]

$r=1-\frac{\mathrm{i} \alpha}{k_{0}^{2}-k^{2}+\mathrm{i} \Gamma}$,

where $\alpha$ is a coupling constant describing the surface mode - photon coupling, $I$ is the damping of the mode and $k$ is the in-plane wave number of the incoming 
photon field. $k_{0}$ is the wave number of the freely propagating surface mode. The damping $\Gamma$ (which corresponds to the width of the ATR resonance) can be decomposed into a radiation damping $\Gamma_{\mathrm{rad}}=\alpha / 2$ (due to the coupling to the photons [19]) and an intrinsic (dissipative) part $\Gamma_{\text {int, }}$ such that $\Gamma=\Gamma_{\text {rad }}+\Gamma_{\text {int }}$. It should be emphasized that Eq. (1) does not contain any information about the nature of the layer system carrying the surface mode under investigation. It is generally valid for optical surface (or interface) modes and describes the changes in amplitude and phase an incoming wave experiences upon reflection.

As the angle of incidence, and hence $k$, is varied, a loop-like orbit results for $r(k)$ in the complex plane. This is plotted in Fig. 1a, the corresponding Lorentzian dip in the reflected intensity is shown in Fig. 1b. Its width depends on the kind of surface mode it represents and can range from less than $10^{-4}$ degrees (angle of incidence) for modes in dielectric planar wave guides to a few degrees in systems which are more strongly damped.

The dip position represents the surface mode wave number and depends sensitively on the physical properties of the system under consideration.

It has been shown before how to utilize this fact for imaging the lateral strucutre of the layer system $[11,20,21]$. The sample is illuminated at an angle of incidence at which parts of the sample are at resonance. Imaging the reflected light onto a camera chip, one obtains an image in which the resonant parts of the sample appear dark, while others appear bright. The narrower the resonance, the higher the image contrast. For instance, a structured $3 \mathrm{~nm}$ film of $\mathrm{SiO}_{x}$ on a silver surface yields a $100 \%$ (dark/white) contrast image when the surface plasmon resonance on the silver surface is used [11], and even a few Angstroms of thickness modulation are sufficient for obtaining reasonable images [20]. The lateral resolution is determined basically by the decay length of the surface mode used, which is inversely proportional to the resonance width and of the order of several microns [21]. Although several modifications of this type of surface microscopy have been employed [22-24], phase information has not yet been obtained in the imaging process.
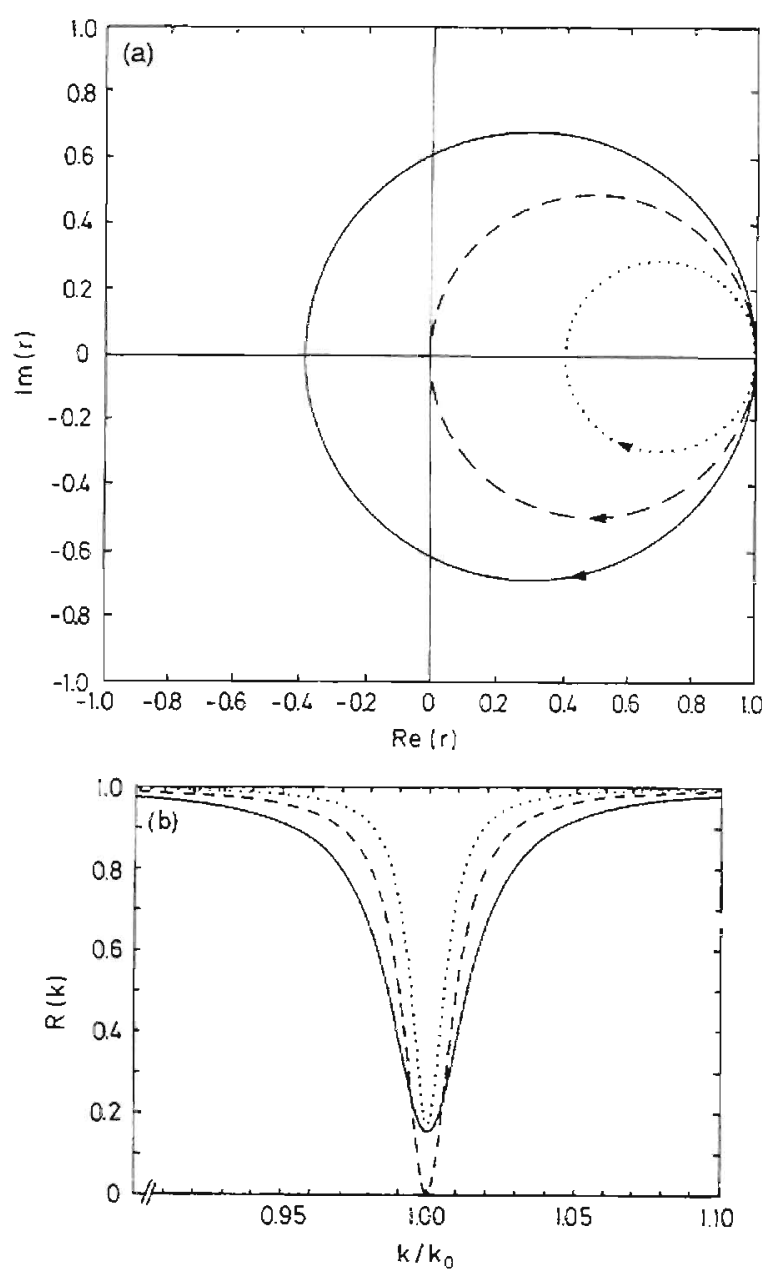

Fig. 1. (a) Orbits of $r(k)$ in the complex plane according to Eq. (1) for $\Gamma_{\mathrm{rad}} / \Gamma_{\text {int }}=0.42$ (dotted), 1.00 (dashed), 2.27 (solid). The arrows indicate increasing $k$. (b) Plot of the reflectivity $R=|r(k)|^{2}$ versus $k$ for the same conditions as in (a).

\section{Experiment}

For the purpose of the present paper, it is important to note that each surface mode can be excited only by light of a particular linear polarization state (either $\mathrm{s}$ or p). Consequently, the photons of the polarization which couples to the surface mode under consideration experience the attenuation and phase shift discussed above, while the photons of the other polarization remain unaffected. This offers the opportunity of using the latter as a reference beam in order to detect the phase shift induced by the excitation of the surface mode and thus get additional information about the system to be imaged. In what follows we will describe how the $s$ and $p$ wave can be brought to interference 


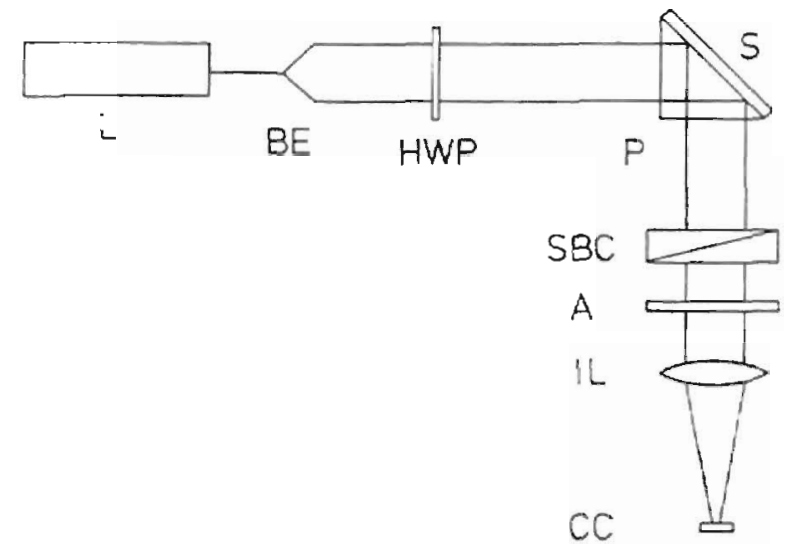

Fig. 2. Experimental setup. L: laser, BE: beam expander. HWP: half wave plate, P: prism, S: sample, SBC: Soleil-Babinet compensator, A: analyzer (polarization sheet at an angle of 45 degrees), IL: imaging lens, CC: camera chip.

with each other and how the phase information can be extracted from the image.

The principle of operation is to illuminate the sample with a well characterized mixture of $s$ and p polarized light and to detect the state of polarization of the reflected light in the vicinity of a surface mode resonance. This can be done using the setup depicted in Fig. 2. After being expanded and spatially filtered, the laser beam passes a half wave plate (HWP) which serves for adjusting the relative intensity of the $s$ and $p$ radiation without affecting their relative phase. The prism is used to couple the beam to the sample. After being reflected from the sample, the beam passes a Soleil-Babinet compensator (SBC) which induces an adjustable phase shift between the $s$ and $p$ wave. Finally, an analyzer which is at 45 degrees with respect to the plane of incidence projects the electric fields of both waves onto the same direction in order to make them interfere with each other. Only when the $s$ and $p$ wave, after reflection from the sample, have equal amplitude and a relative phase equal to $\pi$, the projection of the electric field onto the analyzer direction will vanish and the radiation will be completely blocked. A slight change in the relative phase will give rise to a finite intensity passing the analyzer.

\section{Results and discussion}

To demonstrate the phase measurement, we measured the complex reflection amplitude in the vicinity

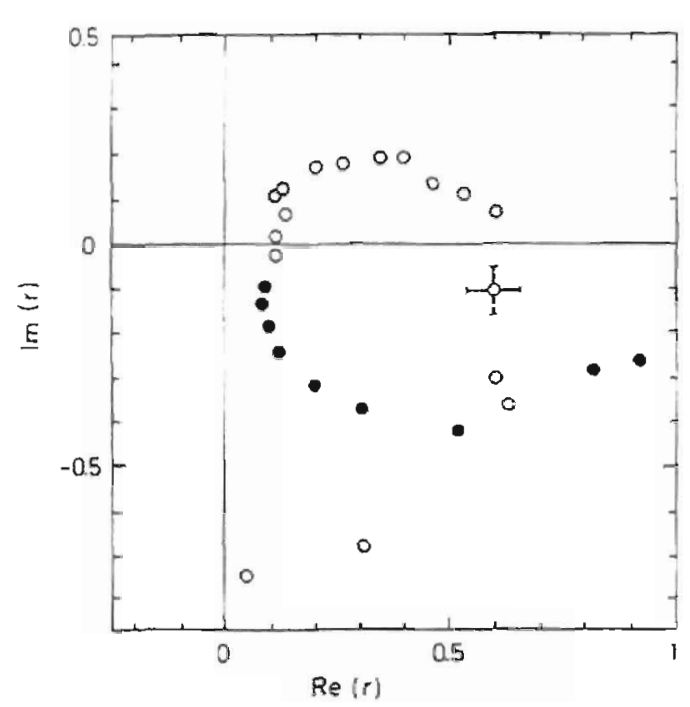

Fig. 3. Orbit of $r(k)$ in the complex plane for the surface plasmon resonance on a $51 \mathrm{~nm}$ thick gold film. Full circles: $k<k_{0}$. Open circles: $k>k_{0}$. The phase of $r$ has been gauged so a 10 give zero at resonance. One representative error bar is given.

of a surface plasmon resonance on a $51 \mathrm{~nm}$ thick gold film. For different angles of incidence, the HWP and the SBC were adjusted so as to make the transmitted intensity go to zero. From the sel of positions of the HWP and SBC obtained in this way, the orbit of the complex reflection coefficient can be derived. This is plotted in Fig. 3, with the phase set to zero at resonance. In the course of the orbit, the angle of incidence is changed by approximately 6 degrees. The orbit directly resembles the loops in Fig. 1a, with the difference in shape stemming from the fact that both the $s$ and $p$ wave experience an additional, monotonously changing phase shift as the angle of incidence is varied, which has been neglected in the derivation of the above formula. By comparison with Fig. 1a, one can see that $\Gamma_{\text {rad }}<\Gamma_{\text {int }}$ in our sample, which could not be judged without the phase information. Making use of this information, one can determine all parameters in Eq. (1) from the width and minimum reflectivity of the ATR resonance. It should be noted that close to the resonance, the phase of the reflected light changes quite rapidly when the angle of incidence is changed. This gives rise to anomalously large Cros-Hänchen effects, which will be elaborated on in a forthcoming paper.

When the SBC is set to a particular phase shift and the reflected light is used for imaging the sample, those, and only those, parts of the sample appear 
dark which correspond to this phase shift. One thus has a direct phase contrast in the image of the sample, which provides additional information about the sample properties. As a demonstration, Fig. 4 shows images of a structure of coloration in a $\mathrm{WO}_{3}$ film on a silver surface. The film had a thickness of $340 \mathrm{~nm}$ so as to support an optical TE waveguide mode, which was used for the imaging here. The $\mathrm{WO}_{3}$ was photochromically colored by illumination with ultraviolet light through a grating. The resulting structure $a b-$ sorbs only weakly, the absorbance of a photochromically colored $340 \mathrm{~nm}$ thick film being only a few percent $[25,26]$. Depending on the positions of the HWP and the SBC, the absorbing parts appear dark Fig. 5a or bright Fig. 5b. In either case, the relative phase between $\mathrm{s}$ and $\mathrm{p}$ wave is $\pi$, but the intensity of the reference wave is different for the two images. Furthermore, whith an intermediate value of the $s$ wave amplitude, it is possible to make the contrast between colored and uncolored parts vanish, as in Fig. 4c. As a consequence, the boundaries between the regions, where the state of coloration is intermediate, are emphasized appearing as dark lines now.

One should bear in mind that without taking advantage of the phase contrast, structures in the absorbance of a sample (as in our experiment) are not easily imaged, unless much care is taken to provide critical coupling for the surface mode used (i.e., $\Gamma_{\text {rad }} \approx \Gamma_{\text {int }}$ ). This is not always easy to achieve, for instance when different regions of a (structured) sample need different coupling conditions. Furthermore, with the aid of the phase information, the contrast between particular objects of a sample can be selectively hidden while enhancing the contrast to others, as shown in Fig. $4 \mathrm{c}$. This might be very useful

when complex structures such as integrated optical devices are to be imaged.

Furthermore, the method is not limited to the usual ATR imaging scheme.

Optically nonlinear films and surfaces are routinely characterized using second harmonic generation (SHG) [27-29]. If one utilizes a surface mode resonance to enhance the fundamental incoming beam amplitude by ATR, one gets appreciable SHG only from the polarization which couples to the resonance. In the spirit of the experiments described above, one can use a second-harmonic beam of different polarization as a reference, which may be generated
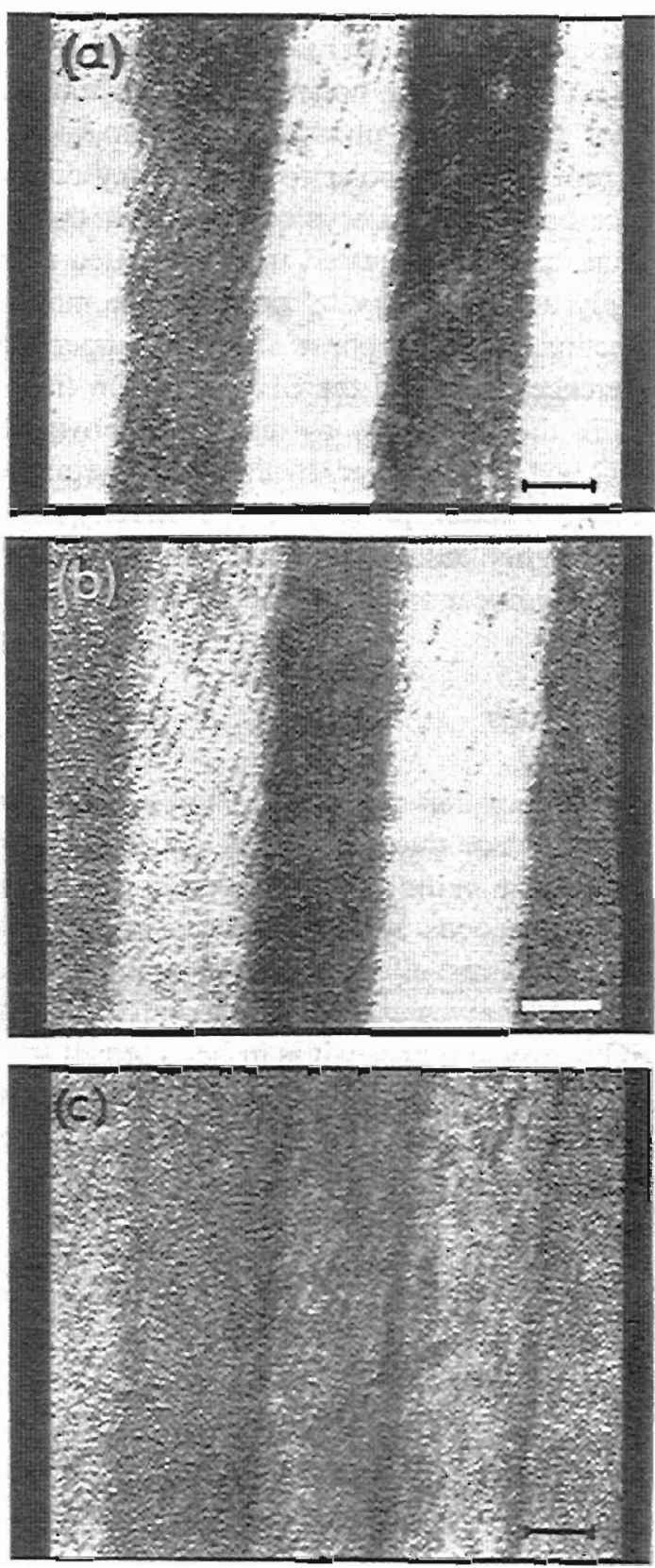

Fig. 4. Images of a slightly absorbing structure in a $340 \mathrm{~nm}$ thick $W_{3}$ film, produced by illumination with ultraviolet light through a grating. A waveguide resonance was used for the imaging. The relative phase between the $s$ and $p$ wave is $\pi$, but the $s$ wave amplitude is different in the three images; (a) absorbing parts dark, (b) absorbing parts bright, (c) homogeneous parts grey, transition regions dark. The length of the bar is $100 \mu \mathrm{m}$. 
by putting a type I phase matched nonlinear crystal into the incoming beam. For instance, when surface plasmons are used for the resonance enhancement of the SHG, one takes a p polarized fundamental pump beam for the surface plasmon excitation, with the crystal providing an s polarized (frequency doubled) reference beam. The latter is collinear with the pump beam and can be attenuated in a controlled manner by simply tuning the crystal out of phase matching. In reflection, one can phase shift and superimpose the reference beam and the SHG radiation from the sample in the same way as discussed above, using the SBC and the analyzer. In this way, the phase of the SHG generation process can be directly imaged. which is highly desirable for the characterization of optically nonlinear materials and devices [30,31].

\section{Conclusions}

We have shown how to obtain phase information in surface resonance microscopy. As a demonstration, a subtle structure in the optical propertics of a topologically homogeneous system has been imaged, using the various contrast effects provided by the technique. Aside from the given example concerning the imaging of SHG conversion phase, it is to be expected that the reconstruction of the actual sample structure from its images can be improved using the phase information together with appropriate image processing.

\section{Acknowledgements}

We are indebted to W. Knoll and J.R. Sambles for many stimulating discussions and $K$. Dransfeld for contributing precious devices to our equipment. This work has been supported by the Zentrum II Energieforschung, Konstanz.

\section{References}

[1] W.H. Weber. Phys. Rev. Ler. 39 (1977) 153.

[2] B. Rothenhäusler, J. Rabe, P. Korpiun, W. Knoi:, Surf. Sci. 137 (1984) 373
[3] A. Taddjeddine, G. Piazza, M. Costa, Europhys. Lett. 5 (1988) 87

[4] M.D. Tillin, J.R. Sambles, Surf. Sci. 237 (1990) 181.

[5] S. Herminghaus, P. Leiderer. Appl. Phys. Let. 54 (1989) 99.

[6] H.E. deBruijn, R.P. Kooyman, J. Greve, Appl. Optics 29 (1990) 1974.

[7] Y. Levi. M. Jurich, J.D. Swalen, J. Appl. Phys. 57 (1985) 2601

[8] S. Herminghaus, P. Lxiderer, Appl. Phys. Ler. 58 (1991) 352.

(9) HE. deBruijn. R.P. Kooyman. J. Greve, Optics Comm. 82 (1991) 425 .

[10] U. Albrecht, A. Otto, P. Leiderer, Phys. Rev. Lett. 68 (1992) 3192.

[11] W. Knoll, Mat. Res. Soc. Bulleuin XVI (1991) 29.

[12] S. Herminghaus, B.A. Smith. J.D. Swalen. J. Opt. Soc.Am B 8 (1991) 2311.

[13] S. Herminghaus, D. Boese, D.Y, Yoon, B.A. Smith, Appl Phys. Lett. 59 (1991) 1043

[14] J.D. Swaten, J. Molec, Electron. 2 ( I986) 155.

[15j G. Zhang, K. Sasaki, Appl. Optics 27 (1988) 1358.

[16] C.A. Helm, W. Knoll, J. Israelachvili, Proc. Nat. Acad. Sci USA 88 (1991) 8169.

[17] G. Panayotou, M.D. Waterfield, P. End. Current Biology 3 (1993) 913.

[18] J.R. Sambles, G.W. Bradbery, Fuzi Yang, Conternp. Phys. 32 (1991) 173.

[19] S. Herminghaus, M. Klopfleisch, H.J. Schmidt, Optics Lett 19 (1994) 293.

[20] W. Hickel, D. Kamp. W. Knoll, Nature 339 (1989) 186

[21] H. Hickel, W. Knoll, Acta Metall. 37 (1989) 2141.

[22] M. Specht J.D. Pedamig, W.M. Heckl, T.W. Hănsch, Phys Rev. Lett 68 (1992) 476

[23] A. Nemerz, H. Knobloch, W. Knoll, Thin Solid Films 226 (1993) 48 .

[24] U. Fernandez. T.M. Fischer, W. Knoll. Optics Comm. 102 (1993) 49

[25] C. Bechinger, G. Oefinger, S. Herminghaus, P. Leiderer, J. Appl. Phys. 74 (1993) 4527.

[26] C. Bechinger, S. Herminghaus, P. Leiderer. Thin Solid Films 239 (1994) 156.

[27] Y.R. Shen, Nature 337 (1989) 519

[28] G.L. Richmond. Electroanalytical Chemistry 17 (1991) 175

[29] M. Eich, A. Sen, H. Looser, G.C. Bjorklurd, J.D. Swalen. R. Twieg. D.Y. Yoon, J. Appl. Phys. 66 (1989) 2559

[30] H.W.K. Tom, T.F. Heinz. Y.R. Shen. Phys. Rev. Lea. 51 (1983) 1983.

[31] G. Berkovic, E. Shvartsberg, Appl. Phys. B 53 (1991) 333 . 